\title{
Effect of activity level after a posterior-stabilized TKA on the relative bone mineral density measured on standard radiographs in periprosthetic tibial bone: a five-year follow-up study
}

\section{Yong Liu}

Sun Yat-sen University First Affiliated Hospital

\section{Peiheng He}

Sun Yat-sen University First Affiliated Hospital

\section{Xing Li}

Sun Yat-sen University First Affiliated Hospital

\section{Yongheng Ye}

Sun Yat-sen University First Affiliated Hospital

Minghao Liu

Sun Yat-sen University First Affiliated Hospital

\section{Weizhi Chen}

Sun Yat-sen University First Affiliated Hospital

\section{Dongliang XU ( $\nabla$ dddrxu@163.com)}

Sun Yat-Sen University https://orcid.org/0000-0002-0927-5778

Research article

Keywords: TKA, BMD, UCLA, Activity level, Rehabilitation

Posted Date: November 24th, 2020

DOI: https://doi.org/10.21203/rs.3.rs-37186/v3

License: (9) (i) This work is licensed under a Creative Commons Attribution 4.0 International License. Read Full License 


\section{Abstract}

Background: The aim of this study was to evaluate the effect of activity level after a posterior-stabilized TKA on the relative bone mineral density measured on standard radiographs in periprosthetic tibial bone.

Methods: A retrospective review identified 110 patients (110 knees $₫ 20$ men/90 women) who underwent PS TKA with 5-year follow-up. Patients activity level was evaluated by University of California Los Angeles (UCLA) activity score, and the relative BMD in periprosthetic tibial bone was measured by Image software on anteroposterior X-ray images. Clinical assessments included Western Ontario and McMaster Universities (WOMAC), Knee Society score, VAS score and UCLA activity score. Nonlinear regression analysis was used to assess the impact of activity levels on periprosthesis bone density.

Results: During 5-year follow-up period, the bone density in the medial, lateral and distal areas decreased compared with that before surgery $(p<0.0001)$. There was a U-shaped distribution between UCLA activity rating and rBMD loss, with the lowest rBMD loss when the UCLA activity score was between 6-8 at 1 and 3 years. The curve fitting of UCLA activity level and $\mathrm{rBMD} \%$ showed there was a parabolic relationship between UCLA activity level and rBMD\% at 1 and 3 years after surgery $(P<0.001, P=0.001)$, while there was no significant relationship between UCLA activity level and rBMD\% at 5 years after surgery $(P=0.436)$.

Conclusions: We found that activity level had a significant effect on radiographic measurements of BMD at early years after surgery and moderate activity was associated with a minimal reduction in proximal tibial BMD.

\section{Background}

An increasing number of patients with end-stage osteoarthritis are receiving treatment with total knee arthroplasty (TKA), which is a successful procedure for alleviating pain, improving functional ability, and enhancing quality of life[1]. Bone mineral density (BMD) is an indicator of bone quality and reflects the material properties, bone metabolism and risk of fracture[2, 3]. Postoperative changes in the periprosthetic bone density in the tibia are closely related to the outcomes of TKA[4, 5]. Several studies[69] have reported a reduction in BMD in the proximal tibia after TKA, which can cause the subsidence of some components, especially of the tibial plateau, and increase the risk of prosthetic loosening and further revision.

Dual X-ray absorptiometry (DXA) is the gold standard clinical method used to evaluate BMD, but it is not used in routine examinations after TKA due to not only the absence of this technology in many hospitals with limited resources but also the high cost of the technology. Several studies have reported that the grayscale values measured on standard radiographs are closely related to BMD and can reflect changes in $\operatorname{BMD}[2,10]$. Hernandez-Vaquero et al[10] revealed a method based on digital radiological densitometry to evaluate bone density using X-ray images. They measured the grayscale values as the relative BMD (rBMD) and validated that the consistency between the true BMD measured by DXA and the rBMD 
measured by this technique was approximately 0.72 to 0.92 . Therefore, this technique could be used as an alternative to DXA to assess changes in BMD after TKA.

Physical activity is a critical part of functional recovery of the knee joint after TKA. Oktas et al[11] concluded that a rehabilitation program for strengthening the muscles around the hip and knee joints was quite important to the success of TKA. However, Kilgus, DJ et al[12] reported that a high level of physical activity was correlated with increased osteoporosis-related prosthetic loosening in patients after total hip replacement. Ponzio et al[13] revealed that increased postoperative activity was associated with an increased risk of revision. Thus, the optimal level of physical activity for patients after TKA is unclear. There is no consensus on the intensity of rehabilitation and physical activity that should be performed after surgery. The aim of this study was to evaluate the effect of activity level after a posterior-stabilized TKA on the relative bone mineral density measured on standard radiographs in periprosthetic tibial bone.

\section{Methods}

\section{Patients}

110 patients with a diagnosis of knee osteoarthritis (Kellgren-Lawrence classification III or IV) from January 2010 to December 2015 were enrolled in the research. These patients accepted unilateral primary cemented TKA by the same surgeon (Professor Xu) approximately 1 week after admission in the Department of Joint Surgery of the First Affiliated Hospital of Sun Yat-Sen University. A posteriorstabilized prosthesis (DePuy Synthes, P.F.C. Sigma, Warsaw, IN, USA) was implanted for TKA. These patients were followed up for up to 5 years, and they returned to the hospital 1 week, 1 year, 3 years, and 5 years after the surgery for full-length X-rays of both lower limbs, also to evaluate clinical function score (WOMAC score and KSS score) and physical activity level score (UCLA activity score). X-rays were used to evaluate postoperative prosthesis position and measure rBMD based on X-ray. This study was approved by the Medical Ethics Committee of The First Affiliated Hospital of Sun Yat-Sen University (code number [2011] 57), and all the procedures followed the principles of the Helsinki Declaration.

\section{rBMD (calibrated grayscale value) measurements}

rBMD was measured with reference to previous literature[2, 10]. The X-ray images at the different time points were collected with the patient in the same standing position and the tibia neutral. Knee flexion was minimized by fixing the tibial tubercle at the lower end of the knee. Rotation was controlled by fixing the heel and the first and second toes. Standard anteroposterior (AP) X-rays obtained during follow-up (one week, 3 months, 1 year, 3 years, and 5 years postoperatively) were saved as JPG files with 255 (8-bit) grayscale and $300 \mathrm{dpi}$ resolution. Ten regions of interest (ROIs) were chosen as the measured regions in tibia: four lateral regions (L1, L2, L3, L4), four medial regions (M1, M2, M3, M4), and two distal regions (D1, D2) (Fig 1). ImageJ, a public domain Java-based scientific image processing and analysis package, was used to measure the mean grayscale value in the established regions of the radiographs. For each designated region, the grayscale value of regions ' $a$ ' and ' $f$ ', representing the surrounding air (assigned ' $a$ ') and the femoral component (' $f$ '), could be regarded as the minimum and maximum grayscale 
measurements in each radiograph for inter-film comparison. To account for variability between follow-up radiographs, the measured grayscale value of each designated region was calibrated by software using the formula:, where $G_{C, R}$ is the calibrated grayscale value, also representing the $r B M D$ in a given region, $G_{R}$ is the mean grayscale value within an $R O I, G_{a}$ is the value of air within the radiograph, and $G_{f}$ is the mean grayscale value of the femoral component[2]. rBMD is the relative ratio, it has no units.

We defined the medial tibial rBMD as the mean values of $M 1, M 2$ and $M 3$, the lateral tibial rBMD as the mean values of $L 1, L 2$ and $L 3$, and the distal tibial plateau as the mean values of D1 and D2, and the tibial rBMD as the mean values of 10 ROIs. The baseline rBMD was measured within 1 week after TKA. The percent change in $r B M D(r B M D \%)$ was calculated by the following equation: $r B M D \%=100 \% *[r B M D$ (baseline)-rBMD (each time)]/ rBMD (baseline).

\section{Clinical outcome evaluation}

All patients were clinically evaluated with respect to knee function using the Knee Society Score (KSS)[14, 15], the Western Ontario and McMaster University Osteoarthritis Index (WOMAC) [15, 16] and visual analogue scale (VAS) score preoperatively and at each follow-up time point (3 months, 1 year, 3 years, and 5 years postoperatively). The level of activity was evaluated using the University of California Los Angeles (UCLA) Activity Rating Scale[17-19]which has ten points: 1) wholly inactive, dependent on others;

2) mostly inactive, restricted to minimal activities of daily living; 3 ) sometimes participate in mild activity such as walking, limited housework or shopping; 4) regularly participate in mild activities; 5) sometimes participate in moderate activity such as swimming and unlimited housework or shopping; 6) regularly participate in moderate activities; 7) regularly participate in active events such as bicycling; 8) regularly participate in very active events such as bowling or golf; 9) sometimes participate in impact sport such as jogging, tennis, skiing, heavy labour; 10) regularly participate in impact sport.

\section{Statistical analysis}

The Shapiro-Wilk test was used to confirm that the data were normally distributed. One-way ANOVA was used to compare clinical scores and rBMD at different time points. Pearson's chi-squared test was performed to clarify the relationship between sex, age, hip-knee-ankle (HKA) angle, BMI and rBMD\% at 1year, 3 years and 5 years. Curve fitting and nonlinear regression were performed to clarify the relationship between the UCLA activity rating and rBMD\% at different time point. Significance was defined as $P<0.05$. All statistical analyses were performed using SPSS 21.0 .

\section{Results}

110 patients had complete clinical and radiological information and were included in the final analysis. Some basic information of patients is shown in Table 1. By the 5-year follow-up examination, the knee score and function score (WOMAC, KSS, VAS score) for each study participant had improved significantly over preoperative scores (Table 2). 
During 5-year follow-up period, the bone density in the medial, lateral and distal areas decreased compared with that before surgery $(\mathrm{p}<0.0001)$. As shown in Figure 2 and Table 3 , compared with the baseline, rBMD of medial side, lateral side and distal side decreased by $3.00 \%, 4.24 \%$ and $1.42 \%$ respectively at 3 months, decreased by $3.51 \%, 5.07 \%$ and $1.42 \%$ respectively at 1 year, decreased by $4.03 \%, 5.57 \%$ and $1.79 \%$ respectively at 3 years, and decreased by $6.68 \%, 9.65 \%$ and $3.29 \%$ respectively at 5 years.

To verify the influence of other factors on rBMD\%, Pearson's chi-squared test was used to examine the association between sex, age, hip-knee-ankle (HKA) angle, BMI and $\mathrm{rBMD} \%$, the results showed that there were no significant association between them (Table 4). To verify the effect of postoperative activity level (UCLA activity scale) on rBMD\%, a line graph of UCLA activity scale and rBMD\% was drawn and the results showed that there was a U-shaped distribution between them $₫$ with the lowest rBMD loss when the UCLA activity score was between 6-8 at 1 and 3 years (Fig 3, Table 5).

To find relation between UCLA activity rating and rBMD\%, the curve fitting has been employed (Fig 4). The curve fitting of UCLA activity rating and $\mathrm{rBMD} \%$ showed there was a parabolic relationship between UCLA activity rating and $r B M D \%$ at 1 and 3 years after surgery $(P<0.001, P=0.001)$, while there was no significant relationship between UCLA activity rating and $r B M D \%$ at 5 years after surgery $(P=0.436)$. The best fit equations obtained are given below as Equation (1) and Equation (2).

$$
\begin{array}{ll}
Y(1 y)=43.16-12.62 X+0.9377 X^{2} & \left(4 \leq \mathrm{X} \leq 9, \mathrm{R}^{2}=0.26\right) \\
Y(3 y)=56-16.73 X+1.254 X^{2} & \left(4 \leq \mathrm{X} \leq 9, \mathrm{R}^{2}=0.36\right)
\end{array}
$$

Where $\mathrm{Y}(1 \mathrm{y})$ and $\mathrm{Y}(3 \mathrm{y})$ are the $\mathrm{rBMD} \%$ at 1 and 3 years respectively, and $\mathrm{X}$ is the UCLA activity rating.

\section{Discussion}

In this study we found that physical activity level was an important factor affecting periprosthesis bone density after TKA and moderate activity was associated with less BMD loss than either low activity and high activity. This research showed that periprosthesis bone density loss was the lowest at activity levels of 6-8, while it increased at both low and high activity levels.

A nonlinear (parabolic) relationship between the UCLA activity rating after TKA and rBMD\% in the proximal tibia suggested that moderate physical activity was beneficial to maintain bone mass, while insufficient or excessive functional exercise aggravated bone loss. This phenomenon is consistent with Wolff's law[20], which indicates that mechanical stress stimulates bone formation, while disuse leads to bone loss. Petersen et al[9] revealed that a decreased load led to rapid bone loss, while an increased load led to a small increase in BMD in the tibial condyles. Gallo, $J$ et al. [21] reported that for man, high levels of physical activity, and a high historical level of physical activity were associated with a higher risk of 
reoperation; for women, the early reoperation was associated with lower physical activity. It seemed that both low and high levels of activity were detrimental to TKA.

The aim of the present study was to evaluate the effect of activity level, determined by the UCLA activity rating, on BMD in periprosthetic bone after TKA and suggest the best activity level to guide clinical rehabilitation postoperatively. Although the rBMD measured on standard X-ray images is not the true $B M D$, Hernandez-Vaquero et al[10] have proven that the relationship between the rBMD measured on standard X-ray images and the true BMD measured by DXA is linear (Cronbach's correlation of 0.72 to 0.92), namely, rBMD could serve as an alternative to BMD.

After TKA, the bone density around the prosthesis decreased, and the medial side decreased more than the lateral side. In fact, the decrease in BMD following TKA had been widely reported and was generally believed to be the result of a biomechanical response to stress shielding of the tibial component[22-26]. Surgery altered the intra-articular environment and osteotomy exposed cancellous bone. The implant is in direct contact with cancellous bone, and the pressure load directly affected cancellous bone, contributing to bone loss. Due to the stress shielding effect, the stress stimulation on the bone around the prosthesis was reduced, and bone tissue absorption occurred, which was manifested as bone loss. Postoperative limb immobilization also contributed to disuse osteoporosis.

The significant influence of activity level on rBMD at 1 and 3 years could be attributed to adaptive bone remodeling and changes in load, as Jaroma, $\mathrm{A}$ et al. reported that tibial metaphyseal periprosthetic bone was remodeled after TKA due to mechanical axis correction [8]. However, the postoperative activity level had no significant effect on BMD after 5 years. Seitz et al[27] found that bone density loss demonstrated a reparation phase and a stabilization phase and that no significant BMD change was observed during the stabilization phase. Hence bone might be in the stabilization phase at 5 years and not be affected by activity level. On the other hand, as time going on, the prosthesis integrated better and better with the surrounding bone, and the bone-prosthesis interface became more and more stable. Therefore, external factors including activity level had little influence on the bone around the prosthesis, so the activity level had no significant effect on BMD at 5 years after surgery. This phenomenon told us that the bone around the prosthesis in the early postoperative period after TKA was greatly affected by external factors, and patients should undertake appropriate functional exercise in the early postoperative period to reduce bone loss, reduce prosthesis looseness and increase the lifespan of prosthesis.

To sum up, there is a decrease in bone density around the prosthesis after TKA, which can be caused by a variety of reasons. Among them, physical activity level is an important factor affecting bone mineral density around the prosthesis. Our study found that both too low and too high activity level had a negative impact on bone mineral density. Only within the appropriate range of activity, bone mineral density loss was minimal. In addition, activity level had an effect on bone mineral density in the early postoperative period, but after 5 years, activity levels had little effect on bone mineral density. Therefore, it was suggested that patients should undergo rehabilitation training with a moderate activity level within early postoperative period. 
There are some limitations to this study. Firstly, the sex distribution was atypical with $82 \%$ females. However, according to relevant statistics, the ratio of male to female knee osteoarthritis is about 1:7, so female subjects are the majority. Secondly, the number of participants in the study was limited. We revealed an interesting relationship between activity level and BMD, but a further study that included more participants is needed. Thirdly the BMD measured on standard X-ray images is a relative value, and while it can reflect the trend of the true BMD, there may be quantitative error with respect to the true BMD.

\section{Conclusion}

Physical activity is important for recovery after TKA; however, there are no consistent guidelines regarding activity intensity. In this retrospective study, we found that activity level had a significant effect on radiographic measurements of BMD at early years after surgery. Moderate activity was associated with a minimal reduction in BMD. Therefore, moderate activity is may be the most appropriate activity intensity for patients with TKA but it is unclear whether a greater activity intensity might be recommended.

\section{Abbreviations}

TKA: total knee arthroplasty, BMD: bone mineral density, rBMD: relative BMD, rBMD\%: the percent change in rBMD, DXA: Dual X-ray absorptiometry, ROls: regions of interest, KSS: Knee Society Score, WOMAC: Western Ontario and McMaster University Osteoarthritis Index, VAS: visual analogue scale, UCLA activity rating : University of California Los Angeles Activity Rating Scale, HKA angle: hip-knee-ankle angle, ANOVA: Analysis of Variance.

\section{Declarations}

-Ethics approval and consent to participate

This study was approved by the Medical Ethics Committee of The First Affiliated Hospital of Sun Yat-Sen University (code number [2011] 57), and all the procedures followed the principles of the Helsinki Declaration.

-Consent for publication

Not applicable

-Availability of data and materials

The datasets used and/or analysed during the current study are available from the corresponding author on reasonable request.

-Competing interests

The authors declare that they have no competing interests 
•Funding

This work was supported by the 5010 Clinical Research Project of Sun Yat-sen University (2010005).

-Authors' contributions

YL and DX conceived the project, YL designed the experiment, collected and analyzed patients' data, and wrote the manuscript, $\mathrm{PH}$ participated in patient follow-up and provided comments, $Y Y, X L, M L$ and $W C$ assisted in patient follow-up. All authors read and approved the final manuscript.

-Acknowledgements

Not applicable

\section{References}

1. Carr AJ, Robertsson O, Graves S, Price AJ, Arden NK, Judge A, Beard DJ: Knee replacement. The Lancet 2012, 379(9823):1331-1340.

2. Small SR, Ritter MA, Merchun JG, Davis KE, Rogge RD: Changes in tibial bone density measured from standard radiographs in cemented and uncemented total knee replacements after ten years' followup. Bone \& Joint Surgery 2013, 95-B:911-916.

3. Sessa G, Costarella L, Puma Pagliarello C, Di Stefano A, Sessa A, Testa G, Pavone V: Bone mineral density as a marker of hip implant longevity: a prospective assessment of a cementless stem with dual-energy X-ray absorptiometry at twenty years. International orthopaedics 2019, 43(1):71-75.

4. Li Y, Ou Y, Zhu Y, Zhao Z, He B, Xu S, Yu H: The predictive significance of bone mineral density on postoperative pain relief in knee osteoarthritis patients after total knee arthroplasty: A prediction model. J Orthop Sci 2020.

5. Bernatz JT, Krueger DC, Squire MW, Illgen RL, 2nd, Binkley NC, Anderson PA: Unrecognized Osteoporosis Is Common in Patients With a Well-Functioning Total Knee Arthroplasty. The Journal of arthroplasty 2019, 34(10):2347-2350.

6. Ritter MA, Davis KE, Small SR, Merchun JG, Farris A: Trabecular bone density of the proximal tibia as it relates to failure of a total knee replacement. Bone Joint $J$ 2014, 96b(11):1503-1509.

7. Winther $N$, Jensen $C$, Petersen $M$, Lind T, Schroder $H$, Petersen $M$ : Changes in bone mineral density of the proximal tibia after uncemented total knee arthroplasty. A prospective randomized study. International orthopaedics 2016, 40(2):285-294.

8. Jaroma A, Soininvaara T, Kroger H: Periprosthetic tibial bone mineral density changes after total knee arthroplasty. Acta orthopaedica 2016, 87(3):268-273.

9. Petersen MM, Nielsen PT, Lauritzen JB, Lund B: Changes in bone mineral density of the proximal tibia after uncemented total knee arthroplasty. A 3-year follow-up of $\mathbf{2 5}$ knees. Acta Orthop Scand 1995, 66(6):513-516. 
10. Hernandez-Vaquero D, Garcia-Sandoval MA, Fernandez-Carreira JM, Suarez-Vazquez A, PerezHernandez D: Measurement of bone mineral density is possible with standard radiographs - A study involving total knee replacement. Acta orthopaedica 2005, 76(6):791-795.

11. Oktas $B$, Vergili $O$ : The effect of intensive exercise program and kinesiotaping following total knee arthroplasty on functional recovery of patients. J Orthop Surg Res 2018, 13(1):233.

12. Kilgus DJ, Dorey FJ, Finerman GA, Amstutz HC: Patient activity, sports participation, and impact loading on the durability of cemented total hip replacements. Clin Orthop Relat Res 1991(269):25-31.

13. Ponzio DY, Chiu Y-F, Salvatore A, Lee Y-Y, Lyman S, Windsor RE: An Analysis of the Influence of Physical Activity Level on Total Knee Arthroplasty Expectations, Satisfaction, and Outcomes. The Journal of Bone and Joint Surgery 2018, 100(18):1539-1548.

14. Insall JN, Dorr LD, Scott RD, Scott WN: Rationale of the Knee Society clinical rating system. Clin Orthop Relat Res 1989(248):13-14.

15. Giesinger JM, Hamilton DF, Jost B, Behrend H, Giesinger K: WOMAC, EQ-5D and Knee Society Score Thresholds for Treatment Success After Total Knee Arthroplasty. The Journal of arthroplasty 2015, 30(12):2154-2158.

16. Walker LC, Clement ND, Deehan DJ: Predicting the Outcome of Total Knee Arthroplasty Using the WOMAC Score: A Review of the Literature. J Knee Surg 2019, 32(8):736-741.

17. Lavernia CJ, Sierra RJ, Hungerford DS, Krackow K: Activity level and wear in total knee arthroplasty. The Journal of arthroplasty 2001, 16(4):446-453.

18. Low AHL, Xin X, Law WG, Teng GG, Santosa A, Lim A, Chan G, Ng SC, Thumboo J: Validation of the UCLA Scleroderma Clinical Trial Consortium Gastrointestinal Tract Instrument 2.0 in English- and Chinese-speaking patients in a multi-ethnic Singapore systemic sclerosis cohort. Clin Rheumatol 2017, 36(7):1643-1648.

19. Zahiri CA, Schmalzried TP, Szuszczewicz ES, Amstutz HC: Assessing activity in joint replacement patients. The Journal of arthroplasty 1998, 13(8):890-895.

20. Enlow DH: Wolff's law and the factor of architectonic circumstance. Am J Orthod 1968, 54(11):803822.

21. Gallo J, Kriegova E, Kudelka M, Lostak J, Radvansky M: Gender Differences in Contribution of Smoking, Low Physical Activity, and High BMI to Increased Risk of Early Reoperation After TKA. The Journal of arthroplasty 2020, 35(6):1545-1557.

22. Lonner JH, Klotz M, Levitz C, Lotke PA: Changes in bone density after cemented total knee arthroplasty: influence of stem design. The Journal of arthroplasty 2001, 16(1):107-111.

23. Soininvaara TA, Miettinen HJA, Jurvelin JS, Suomalainen OT, Alhava EM, Kröger HPJ: Periprosthetic tibial bone mineral density changes after total knee arthroplasty囚one-year follow-up study of 69 patients. Acta Orthop Scand 2004, 75 (5):5.

24. Abu-Rajab RB, Watson WS, Walker B, Roberts J, Gallacher SJ, Meek RM: Peri-prosthetic bone mineral density after total knee arthroplasty. Cemented versus cementless fixation. J Bone Joint Surg $\mathrm{Br}$ 2006, 88(5):606-613. 
25. Prince JM, Bernatz JT, Binkley N, Abdel MP, Anderson PA: Changes in femoral bone mineral density after total knee arthroplasty: a systematic review and meta-analysis. Arch Osteoporos 2019, 14(1):11.

26. Li MG, Nilsson KG: Changes in bone mineral density at the proximal tibia after total knee arthroplasty: A 2-year follow-up of $\mathbf{2 8}$ knees using dual energy x-ray absorptiometry. J Orthopaed Res 2000, 18(1):40-47.

27. P S, P R: CT bone densitometry of the anchorage of artificial knee joints. J Comput Assit Tomogr 1985(9):607-608.

\section{Tables}

Table 1 Patient characteristics

\begin{tabular}{ll}
\hline Characteristic & Mean (SD, range) \\
\hline Age & $66.59(7.41,50-87)$ \\
Sex $(\mathrm{n})$ & 20 men/90women \\
\hline BMI $\left(\mathrm{kg} / \mathrm{m}^{2}\right)$ & $26.61(3.44,18.56-39.54)$ \\
\hline HKA angle & $180.42(1.45,175-185)$ \\
\hline Operative duration (min) & $135.00(36.21,60-270)$ \\
\hline LOS (day) & $11.61(2.91,7-22)$ \\
\hline Kellgren-Lawrence classification & \\
III & 40 \\
\hline IV & 70 \\
\hline
\end{tabular}

* BMI, body mass index; HKA, hip-knee-ankle, after surgery; LOS, length of stay.

Table 2 Clinical evaluation of participants $(\mathrm{N}=110)$

\begin{tabular}{lllllll}
\hline & Preoperative & 3 months & 1 year & 3 years & 5 years & $P$ \\
\hline KSS score & $77.89 \pm 24.28$ & $120.30 \pm 13.42$ & $165.00 \pm 8.56$ & $170.40 \pm 8.86$ & $182.10 \pm 8.76$ & $<0.001$ \\
WOMAC score & $43.85 \pm 13.24$ & $27.05 \pm 11.54$ & $10.05 \pm 3.40$ & $5.50 \pm 2.93$ & $3.39 \pm 1.97$ & $<0.001$ \\
VAS score & $6.78 \pm 1.23$ & $5.63 \pm 1.28$ & $2.31 \pm 1.24$ & $1.35 \pm 0.44$ & $1.20 \pm 0.69$ & $<0.001$ \\
\hline
\end{tabular}

Note: Values are the mean \pm SD. P value is compared between 5 years after surgery and preoperative by $t$ test.

Table 3 rBMD in Lateral, Medial and Distal region at different time point

\begin{tabular}{ccccccc}
\hline & baseline & 3months & 1 year & 3years & 5years & $P$ \\
\hline $\mathrm{N}$ & 110 & 110 & 110 & 110 & 110 & - \\
$\mathrm{L}$ & $120.2 \pm 6.81$ & $115.1 \pm 8.29$ & $114.1 \pm 9.37$ & $113.5 \pm 9.87$ & $108.6 \pm 9.13$ & $<0.0001$ \\
$\mathrm{M}$ & $116.7 \pm 8.23$ & $113.2 \pm 8.43$ & $112.6 \pm 9.60$ & $112.0 \pm 10.88$ & $108.9 \pm 9.37$ & $<0.0001$ \\
$\mathrm{D}$ & $133.9 \pm 8.22$ & $132.0 \pm 7.85$ & $132.0 \pm 7.77$ & $131.5 \pm 7.64$ & $129.5 \pm 7.17$ & 0.72 \\
\hline
\end{tabular}

Note: $\mathrm{N}$ is the number of participants; $\mathrm{L}$ is lateral region; $\mathrm{M}$ is medial region; $\mathrm{D}$ is distal region. 
Table 4 Pearson's chi-squared test for sex, age, hip-knee-ankle (HKA) angle, BMI and rBMD\%

\begin{tabular}{ccccccc}
\hline & \multicolumn{5}{c}{ rBMD $\%$} \\
\cline { 2 - 6 } & Pearson correlation & $P$ & Pearson correlation & $P$ & Pearson correlation & $P$ \\
\hline \multirow{2}{*}{ Sex } & 0.069 & 0.473 & 0.100 & 0.297 & 0.065 & 0.50 \\
Age & 0.159 & 0.097 & 0.045 & 0.639 & 0.001 & 0.995 \\
\hline HKA angle & 0.052 & 0.592 & 0.059 & 0.541 & 0.094 & 0.330 \\
\hline BMI & 0.118 & 0.219 & 0.010 & 0.918 & 0.018 & 0.848 \\
\hline
\end{tabular}

Note: Pearson's chi-squared test was used to examine the association between sex, age, hip-knee-ankle (HKA) angle, BMI and rBMD\%, the results showed that there were no significant association between them.

Table 5 rBMD\% at different activity levels and different time point

\begin{tabular}{ccccccc}
\hline UCLA & \multicolumn{7}{c}{ rBMD\% } \\
\cline { 2 - 7 } activity level & $1 \mathrm{y}$ & $\mathrm{N}$ & $3 \mathrm{y}$ & $\mathrm{N}$ & $5 \mathrm{y}$ & $\mathrm{N}$ \\
\hline 4 & $6.60 \pm 5.19$ & 12 & $8.21 \pm 4.29$ & 14 & $6.62 \pm 1.73$ & 11 \\
5 & $5.30 \pm 5.30$ & 15 & $5.71 \pm 5.87$ & 13 & $7.36 \pm 4.93$ & 16 \\
6 & $0.52 \pm 2.55$ & 22 & $0.63 \pm 3.14$ & 26 & $5.82 \pm 3.54$ & 22 \\
7 & $1.50 \pm 2.98$ & 20 & $-0.22 \pm 3.07$ & 16 & $6.88 \pm 3.97$ & 19 \\
8 & $0.76 \pm 3.46$ & 18 & $2.13 \pm 4.86$ & 19 & $4.76 \pm 4.75$ & 20 \\
9 & $6.09 \pm 4.16$ & 23 & $7.34 \pm 4.30$ & 22 & $7.19 \pm 4.02$ & 22 \\
\hline
\end{tabular}

Note: Values are the mean \pm SD. $\mathrm{N}$ is the number of participants; rBMD $\%=100 \% *$ [rBMD (baseline)-rBMD (each time)]/ rBMD (baseline). A positive value indicates a decrease in rBMD, while a negative value indicates an increase in rBMD.

\section{Figures}




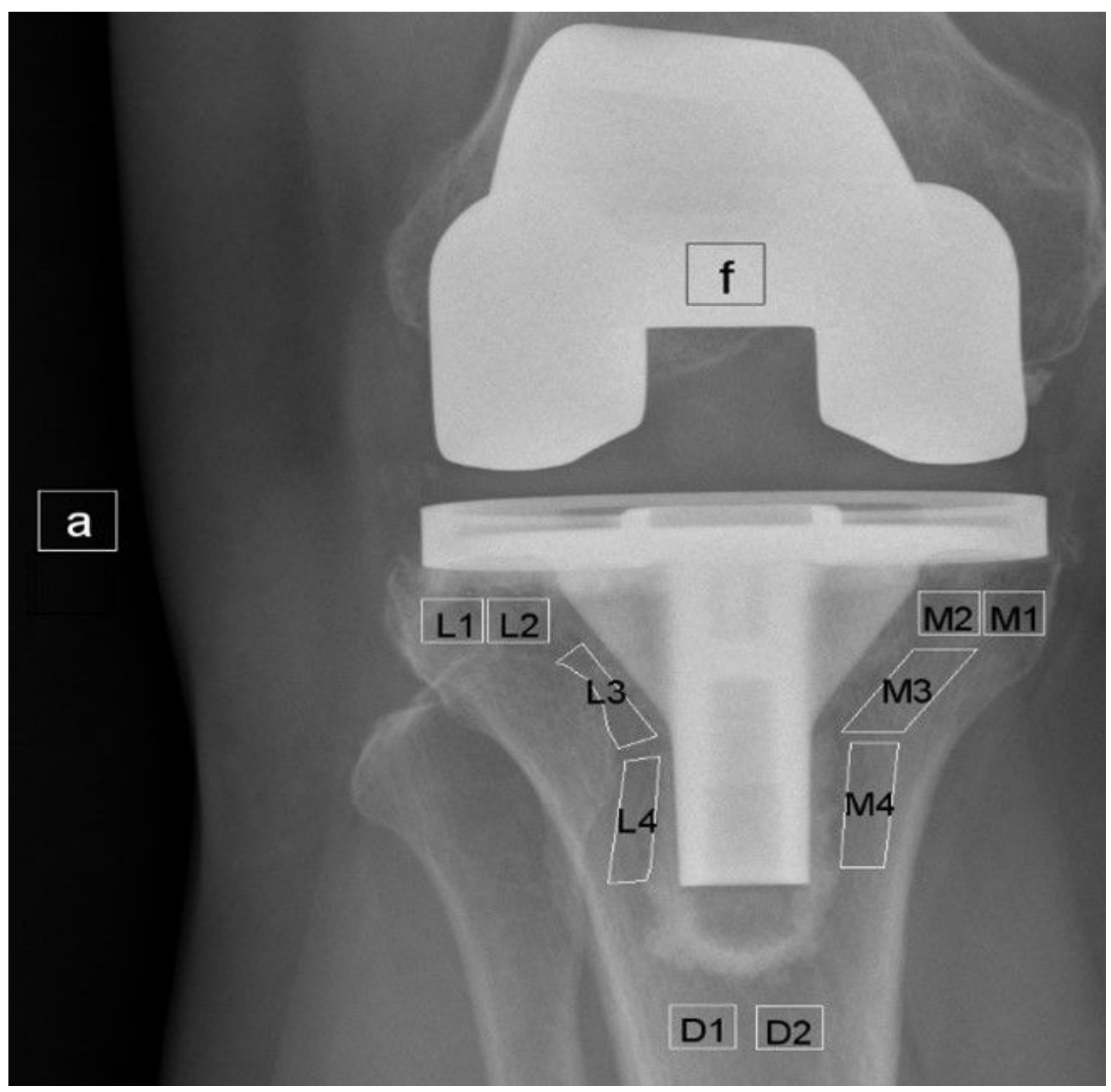

\section{Figure 1}

The rBMD was measured at 10 regions of interest around the tibial component on X-ray film, with 4 medial measurement areas: M1, M2, M3 and M4; 4 lateral measurement areas: L1, L2, L3 and L4; and 2 distal measurement areas: D1 and D2. In the figure above region A and F measure gray values of air and metal of femoral prosthesis respectively to adjust bone density. The measured area avoided cement, cortical bone, and fibula head. The mean value of rBMD around the tibial component was defined as $(\mathrm{M} 1+\mathrm{M} 2+\mathrm{M} 3+\mathrm{M} 4+\mathrm{L} 1+\mathrm{L} 2+\mathrm{L} 3+\mathrm{L} 4+\mathrm{D} 1+\mathrm{D} 2) / 10$. The medial mean rBMD was defined as $(M 1+M 2+M 3+M 4) / 4$. The lateral mean rBMD is defined as $(L 1+L 2+L 3+L 4) / 4$. 


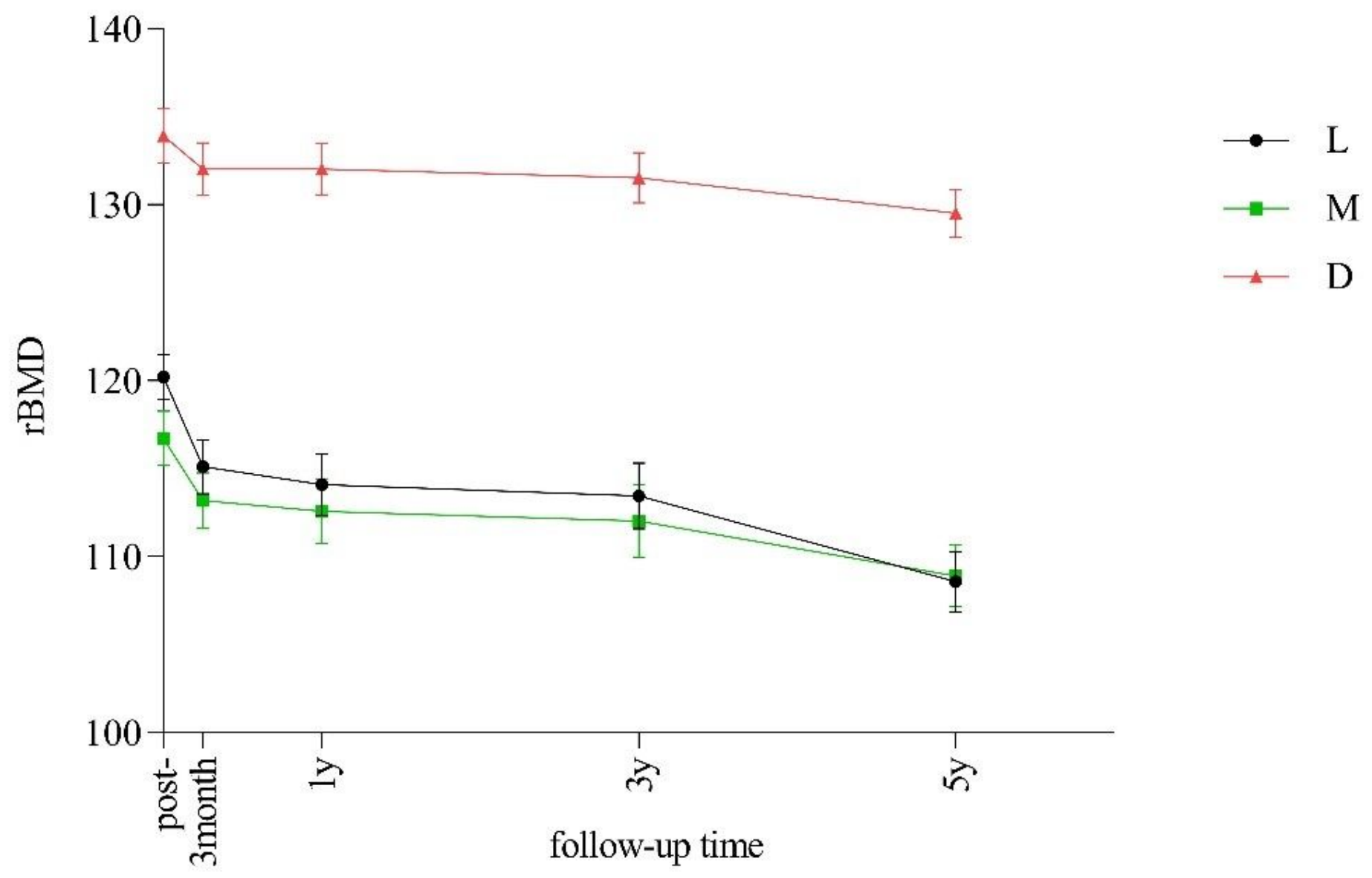

Figure 2

Curves of mean rBMD in medial, lateral and distal regions. rBMD decreased at all time points after surgery compared with baseline. The distal average rBMD of prosthesis was higher than that of medial and lateral average rBMD, while the medial average BMD decreased more than that of lateral average BMD. L囚lateral; M:medial; D:distal. 


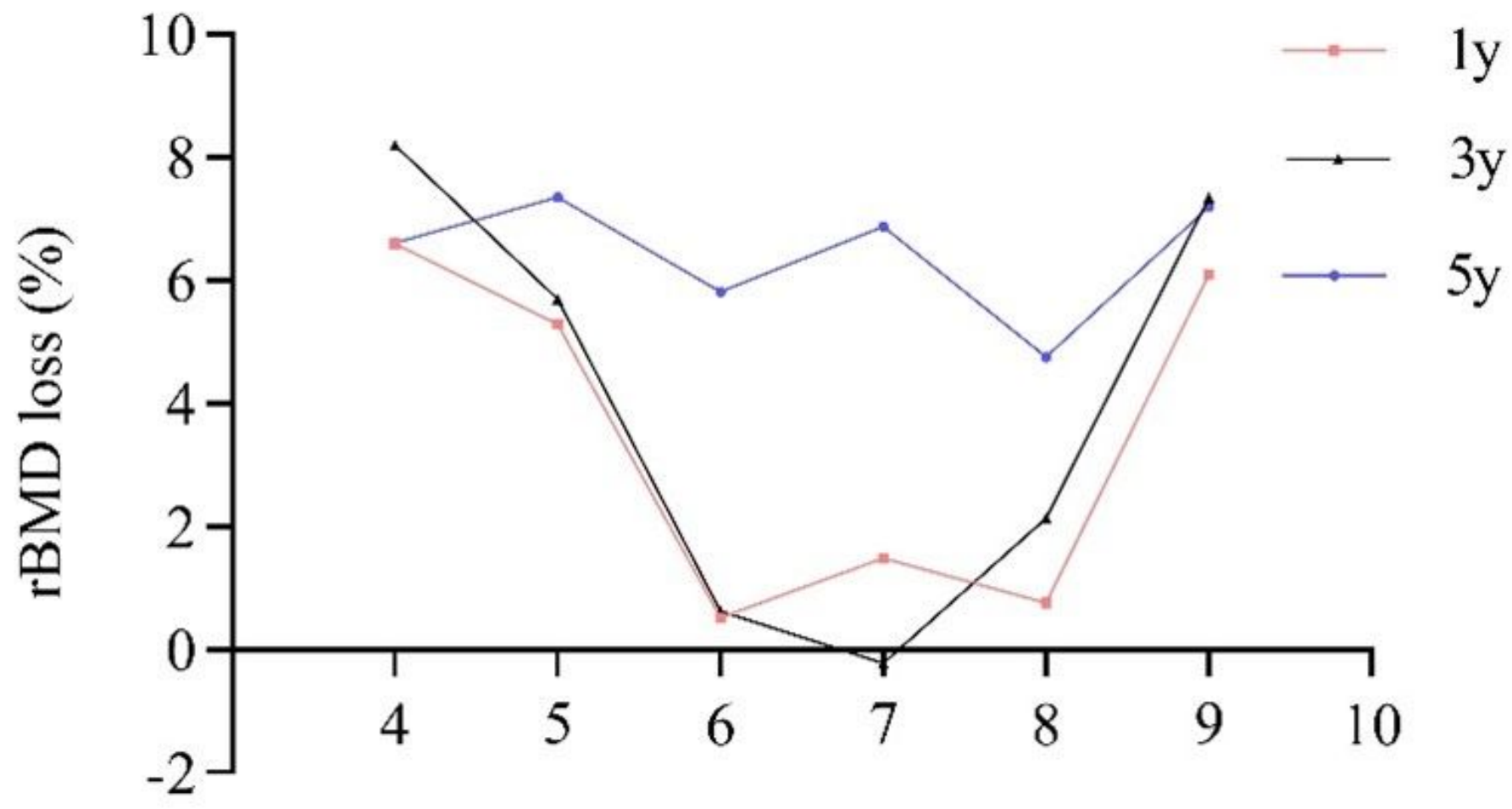

UCLA activity scale

\section{Figure 3}

Relationship of UCLA activity rating with rBMD\%. The rBMD measured at 1 week after surgery was defined as baseline. rBMD\%=(rBMD baseline -rBMD)/rBMD baseline * $100 \%$, indicating the percentage change of rBMD measured after surgery relative to baseline. rBMD \% represents the percentage decrease of $r B M D$, since the bone density basically showed a decreasing trend after surgery. It can be seen from the figure that when UCLA was $6-8$, the rBMD\% was the smallest. There was a U-shaped distribution for them with the lowest rBMD loss when the UCLA activity score was between 6-8 for 1 and 3 years. There was no such relationship at 5 years. 


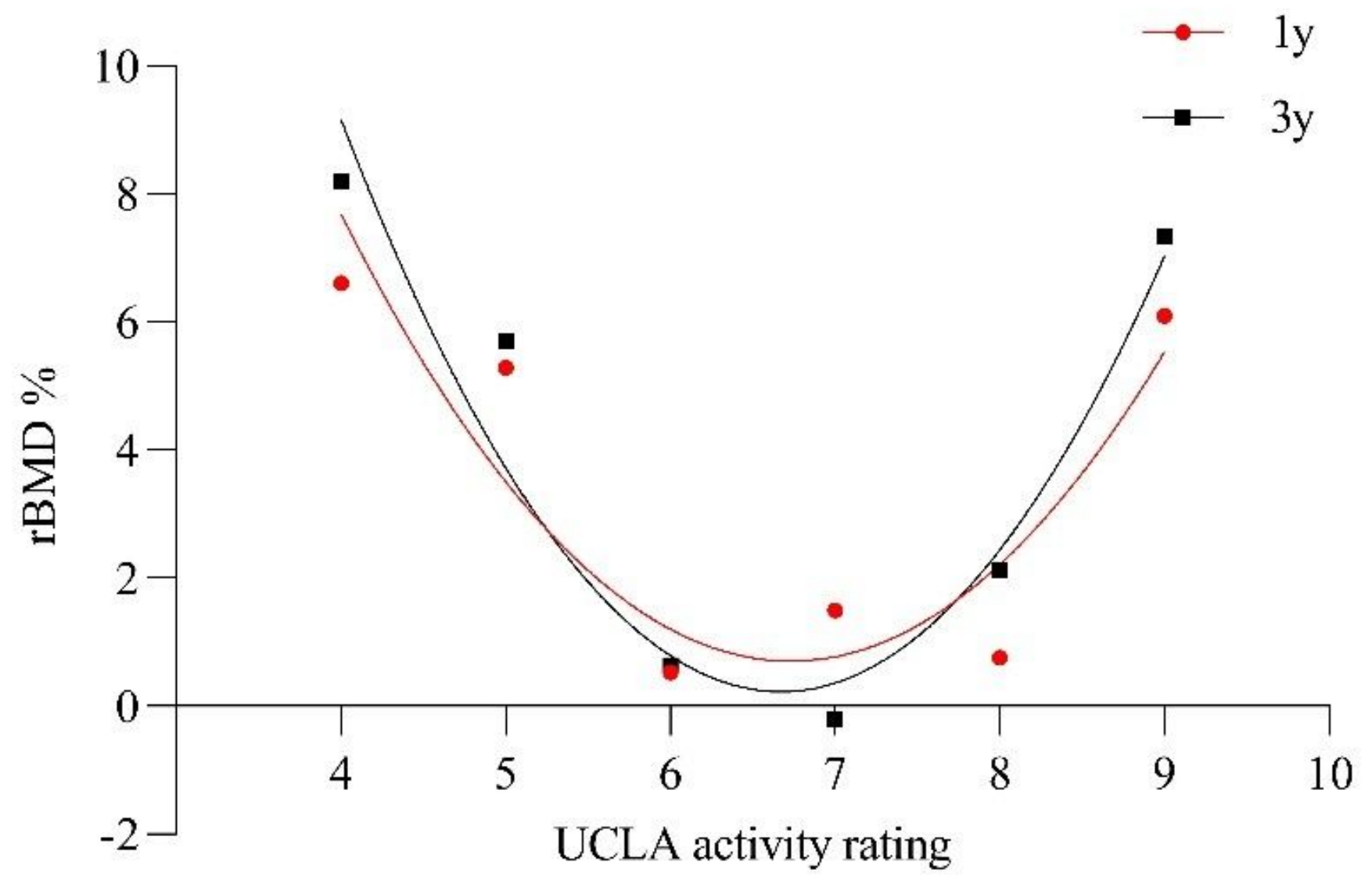

Figure 4

Nonlinear regression model of UCLA activity rating and rBMD\% for measurements at 1 and 3 years. The curve fitting of UCLA activity level and rBMD\% showed there was a parabolic relationship between UCLA activity rating and $r B M D \%$ at 1 and 3 years after surgery $(P<0.001, P=0.001)$, while there was no significant relationship between UCLA activity rating and rBMD\% at 5 years after surgery $(P=0.436)$. This figure showed that postoperative activity level was an important influencing factor of bone density around the prosthesis, and we further discover that the activity level had an effect on the bone around the prosthesis in the early postoperative period, but not in the late postoperative period. Bone density loss around the prosthesis was minimal at activity levels of 6-8. The analysis shows that moderate intensity activity may mitigate against BMD loss around the tibial component. 